\title{
Bursting Through the Blocks in the Human Mind:
}

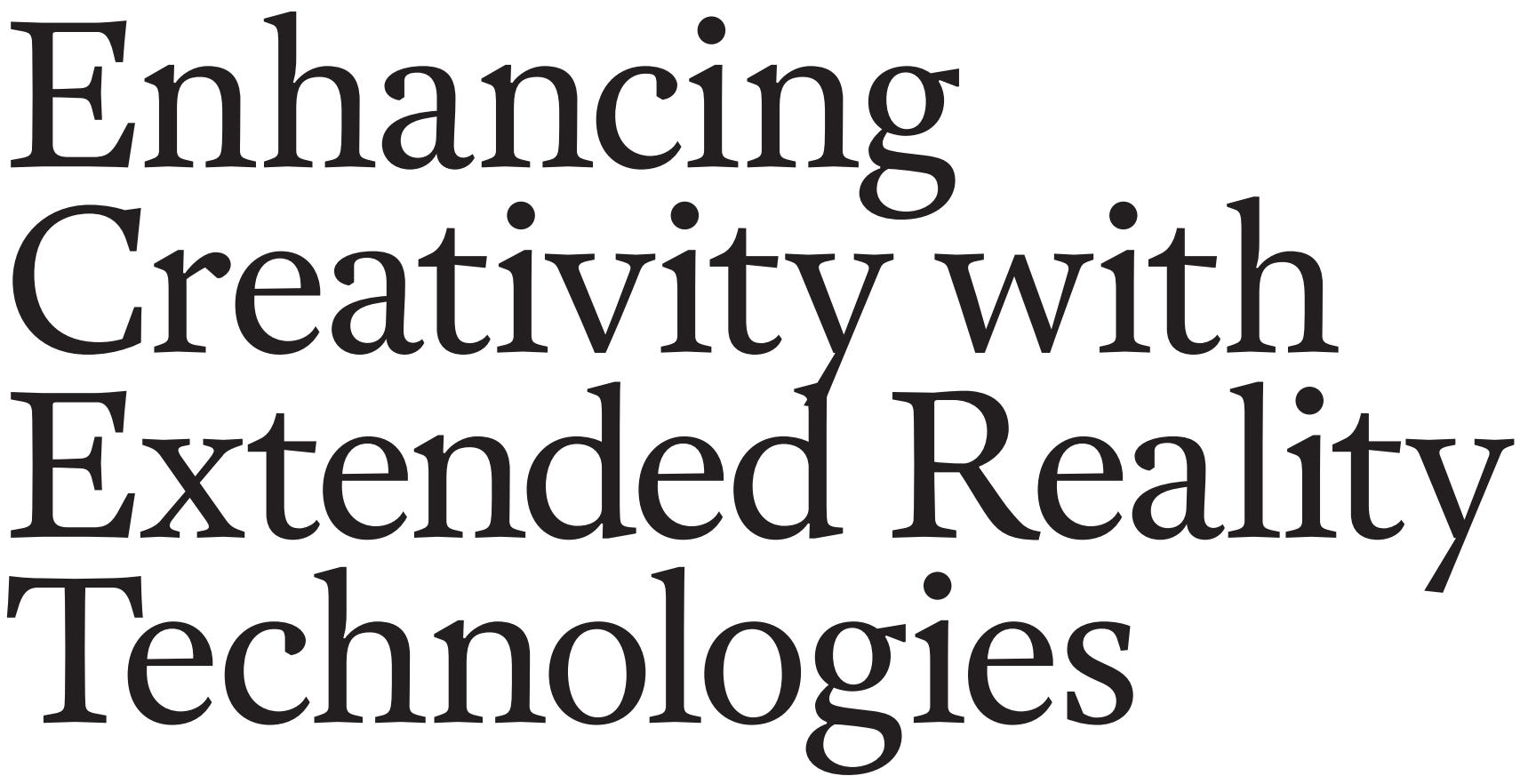

\author{
Insights \\ $\rightarrow$ XR technologies can help \\ remove the barriers in the \\ mind by directly addressing \\ underlying mental \\ mechanisms. \\ $\rightarrow$ Mind-fixation phenomena \\ have distinct subtypes, \\ which can be mitigated \\ using XR technologies by \\ incorporating different \\ features. \\ $\rightarrow$ XR technologies can more \\ strongly support creativity \\ and innovation if their full \\ capabilities can be realized.
}

Encountering barriers in creativity and ideation sometimes feels like hitting a wall, which explains why this metaphor is often used to portray a fixation of the mind [1]. Removing the blocks in the human mind that impede the ideation process is becoming increasingly important, particularly in fields involving innovation and creativity. Humans often build invisible walls in their minds that confine their thoughts in a limited space. These mental walls may result from the faulty integration of previous experiences, aversion to loss, flaws in human cognition, or even pure stereotypes. They impose illusory boundaries when exploring the solution space, producing a narrow mindset. In the ideation process, existing concepts are repeatedly activated and reinforced, thus hampering the generation of new ideas. Breaking through these mind blocks has been a longstanding problem for researchers. As many of these barriers are rooted in inherent mental processes, they are difficult to avoid. Fortunately, rapidly advancing extended reality (XR) technologies have shed new light on this issue by addressing the underlying cognitive and behavioral mechanisms, helping us to avoid pitfalls during the 


\section{Remove Barriers in the Mind}

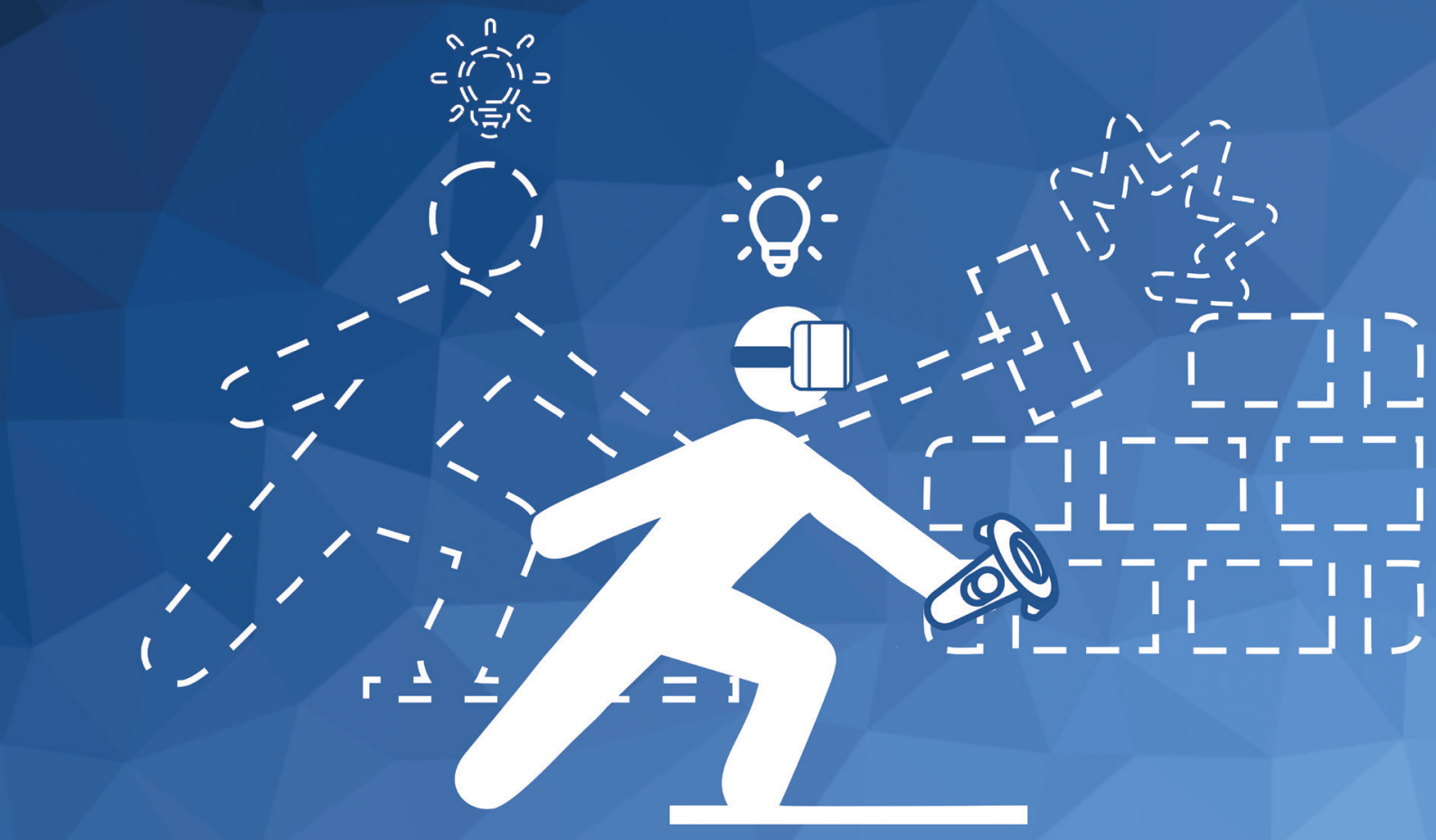

Figure 1. Breaking through the blocks in the mind with XR.

ideation process and enhance our creativity (Figure 1).

\section{MIND FIXATION}

Based on individuals' perception of their own mind-fixation situation and their motivations, Robert Youmans and Tomasz Arciszewski defined three categories of fixation: unconscious adherence, conscious blocking, and intentional resistance [2] (Figure 2). Specifying the driving factors for each of these dimensions will help demonstrate how the features of XR technologies can mitigate each of them and facilitate creativity.

Unconscious adherence refers to the circumstance where individuals are influenced by previously exposed information and fixate their minds on it without awareness. In the most extreme cases, they may focus on features that were presented to be excluded during ideation but remain blind to this situation. The unconscious repetition of preexisting ideas suggests the traits of the following cognitive mechanisms:

- Priming effect: Exposure to previous knowledge can bias people in the subsequent ideation process, leading to an unconscious inclination toward recently observed features.

- Anchoring effect: Humans have a natural tendency to anchor their problem framing based on initial or preexisting information, which can make people view topics with a fixed lens and hence limit the ideation process.

- Loss aversion: This is a natural psychological bias in which people prioritize avoiding loss over other factors. When switching to a new idea implies abandoning the previous effort, there will be a huge psychological burden on people that prevents them from making the change. Thus, it is common for people to seize on early ideas they've put time and energy into and be reluctant to change, even unconsciously. For example, Vimal Viswanathan and Julie Linsey [3] showed that effortconsuming material for ideation, and even imagination using this material, can lead to a higher fixation rate and fewer creative ideas.

- Monotonous stimuli: Human memory is stored in the form of a web of neurons. Activation on a certain memory episode can radiate to the associated neural pathways. Richer stimuli will activate a larger web of neurons and lead to more creative results. However, the reality is often the opposite: The available stimuli are quite monotonous and lead to narrow ideation outcomes.

Conscious blocking, on the other hand, is a situation where individuals are clearly aware of and frustrated by their inability to deviate from fixed ideas. This type of mind fixation has a particularly strong impact in the designer community. In this case, existing strategies and prior knowledge seem to occupy a designer's solution space in a paradoxical way: The harder people try, the more their idea generation processes are jammed, which reveals the role of the interconnected nature of our memory: 
- Interconnected nature of memory: In the web of neurons where humans store their memory episodes, the activity of a piece of information and its accessibility influence each other in a closed-loop process. The recently fired nodes become more active and hence more accessible during memory retrieval, which in turn further increases their chance to be revisited. Thus, in the context of conscious blocking, mind fixation tends to occur when the corresponding neural pathways are overly activated and become much easier to reach than other thoughts. This explains why simply trying harder, or the many intervention methods involving changing the mind or taking a brief rest, will not help; in fact, they worsen the fixation problem. The key is to break the closed-loop reinforcement process.

Intentional resistance, as opposed to involuntary mind fixation, can be characterized by the preference for previously successful methods and the reluctance to change. This category of mind fixation often involves more tangible concerns. Emotional and stereotypical factors can also facilitate mind-fixation behaviors, at both individual and organizational levels:

- Status quo bias: Under this bias, people tend to perceive any deviation from the current situation as a loss. Therefore, unless an idea is evidently proven beneficial, the existing routine will be maintained even though the new thoughts seem to be promising.

- Sunk cost fallacy: This fallacy is a direct result of loss aversion. The unwillingness to "sink" the spent and unrecoverable resources creates strong psychological pressure when facing change, which leads people to mistakenly include the sunk cost for decision making. Such a tendency is stronger when tangible costs are involved, such as budget savings, path reliance, and risk avoidance, particularly at the organizational level. Thus, even when creative ideas are generated, people may choose to stick with existing ones.

- Stereotypes: The stereotypes in the discussion on mind fixation are often led by the incorrect generalization of context-dependent rules or a biased mindset from previous experiences. In both cases, the problem is that people quickly turn down new ideas merely based on their heuristic and are unwilling to investigate further.

The discrepancy between these categories of mind fixation makes mitigating them a challenge. In this context, a new mode of interactionnamely, the rapidly evolving XR technologies - could provide a solution to address underlying mental mechanisms.

\section{XR TECHNOLOGIES}

In the current discussion, XR technologies can be viewed as equivalent to the combination of virtual reality (VR), augmented reality (AR), and mixed reality (MR).

On the reality-virtual scale, VR is at the far end of the virtual side, as this technology involves immersing people in a computer-generated virtual environment. Head-mounted displays (HMDs) and the CAVE automatic virtual environment technique can help create a sense of immersion. An operator's interactions with the virtual world are accomplished by body movements realized via handheld joysticks, gloves, or gesture-recognition devices, similar to our natural ways of expressing emotions and ideas.

$A R$, in essence, is a technology that integrates virtual elements into reality, improving human experiences in the real world. Overlaying virtual elements, particularly the relevant information of an object, such as its parameters and internal structure, has been a popular use of AR, particularly by designers and creativity researchers. As most current AR techniques are mediated by mobile devices, the interaction is often limited to certain areas or objects.

MR blends the virtual and realworld elements in a more balanced way. To make sense of this concept, a popular interpretation is that MR mixes two specific features of VR and AR: a high level of immersion and realness. MR and VR both turn the entire space around users into an interactive environment, whereas MR and AR both blend real-world and virtual elements.

While these three branches of XR each have their own specific features and user scenarios, in the context of mitigating mind fixation, we will discuss XR technologies as a whole. This is because the critical features for enhancing creativity and removing the blocks in the mind, particularly intuitive operation, rich and multimodal stimuli, extendibility, and easy recovery of the ideation process, are shared by all three of these approaches. These features collectively help people to avoid fixation-facilitating mental mechanisms by removing the perceptual, conceptual, and decisionmaking blocks during idea generation (Figure 3).

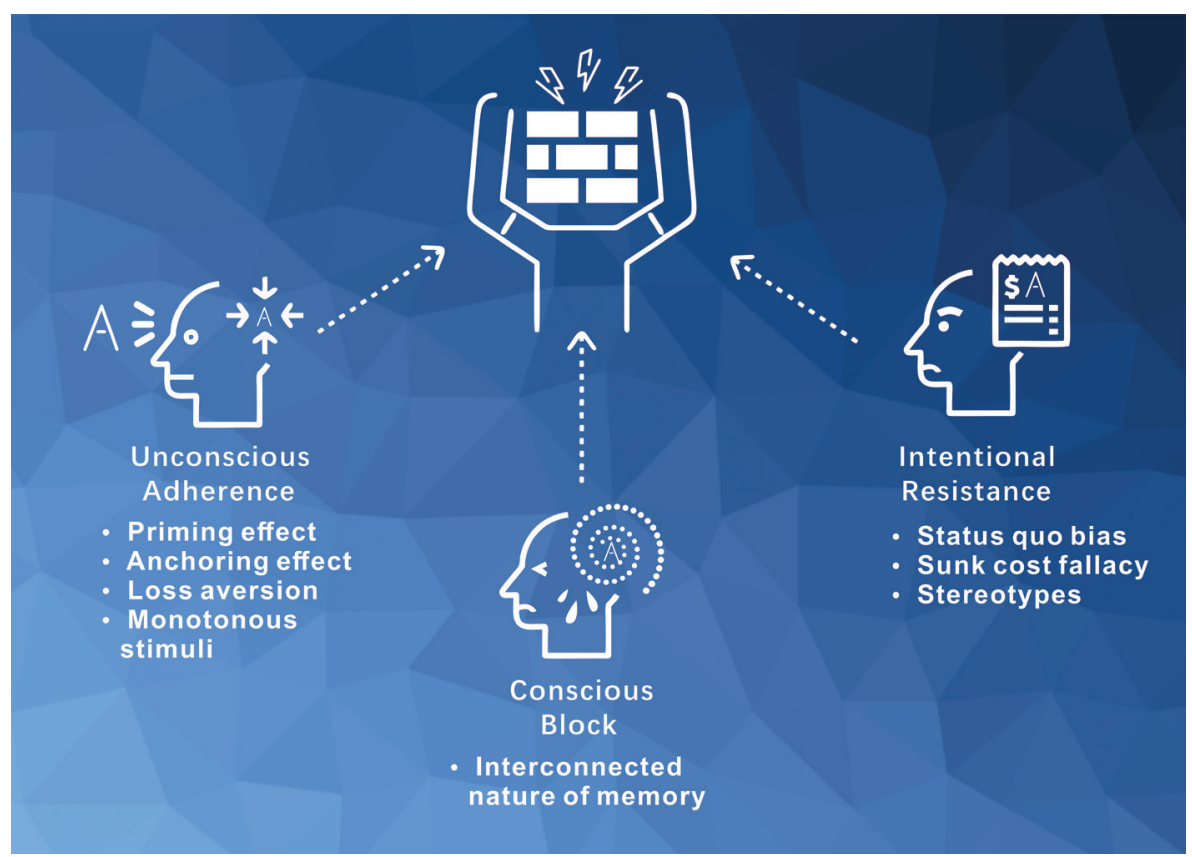

Figure 2. The three types of mind fixation. 


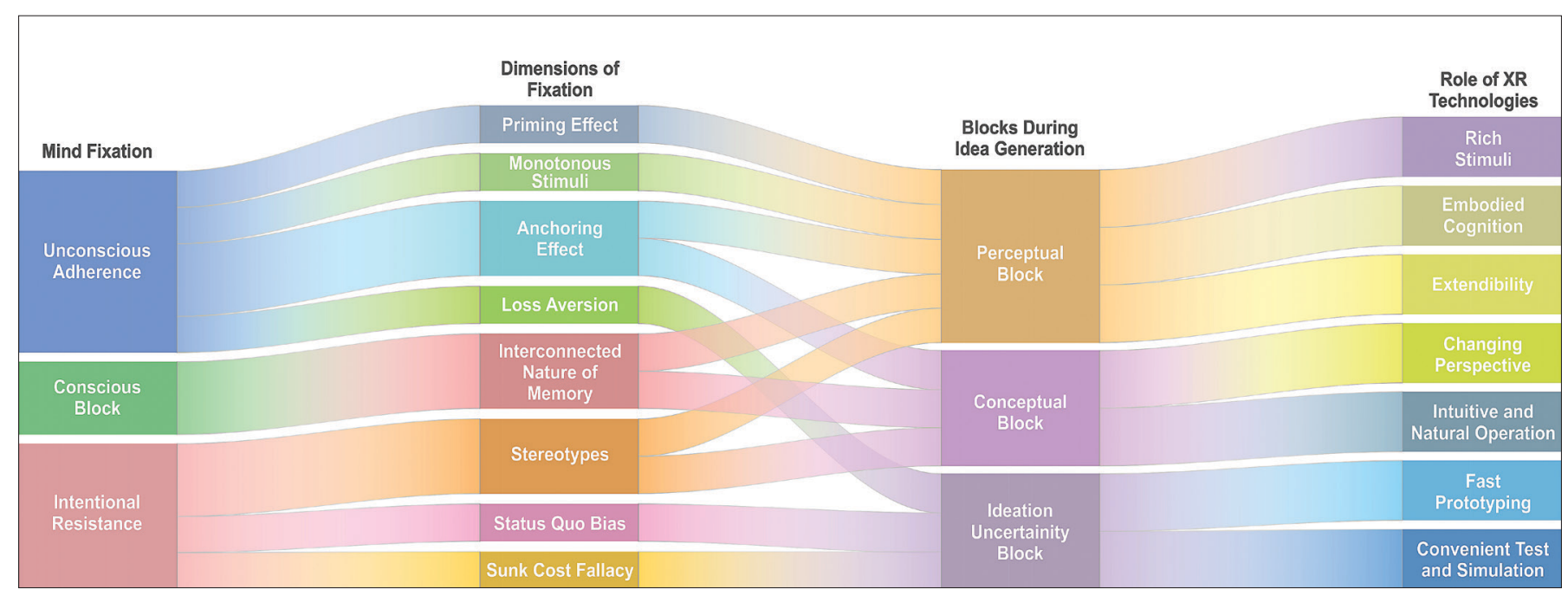

Figure 3. How XR mitigates mind fixation.

Mitigating perceptual blocks. The existence of perceptual blocks causes people to see only what they expect and ignore other opportunities. In mitigating this situation, $\mathrm{XR}$ technologies can extend people's vision in the idea-generation process by providing rich stimuli, recruiting embodied cognition, and integrating other advanced features:

- Rich stimuli. Due to the interconnected structure of memory, a stimulus from an external environment is often required to initiate the knowledge-retrieving process. When perceiving richer stimuli, people can activate a wider range of neural pathways through a web of neurons, access a more diverse pool of knowledge, and explore a broader solution space. This also facilitates people to bring less relevant concepts together, which is a crucial source for innovation. By integrating multisensory modalities and $3 \mathrm{D}$ spatial cognition, current $\mathrm{XR}$ technologies have already outperformed most other competing techniques in providing different types of stimuli and enhancing creativity. For example, in a study by Yang et al. [4], participants were asked to ideate wearable devices either based on a 3D human-body model in an immersive virtual environment or with a 2D-printed human-body image. They concluded that participants in the $3 \mathrm{D}$ condition generated more unique ideas, whereas the participants under the $2 \mathrm{D}$ condition tended to provide highly repetitive ideas that are similar to existing products. This discrepancy in performance was believed to be related to the opportunities of observing the human-body model from multiple perspectives and visualizing the outcomes of using different materials with a digital brush in the virtual environment [4]. Moreover, recruiting nonvisual sensory channels has been a widely recognized strategy in promoting the novelty and creativity of ideas. Thus, XR technologies can significantly enrich the types of stimuli in the design process and help reduce the effect of monotonous stimuli.

- Embodied cognition refers to the phenomenon in which humans' physical perception can influence cognition via a mind-body link. For example, it has been observed that by physically embodying abstract metaphors, such as "being open" or "breaking the wall," designers are able to enhance the novelty and originality of their ideas. Leung et al. [1] also showed that walking freely or walking out of a room during the ideation process, which served as an embodied metaphor of "thinking out of the box," can promote the creativity of designers. This is because the information sensed by the human body intertwines with the ongoing cognitive process and shapes the thoughts. Engaging massive body movement in operation and providing convenient environment settings, XR technologies are ideal media for integrating embodied cognition into the creative ideation process.

- Extendibility. As XR technologies gradually reach their full potential, particularly with the recent breakthrough in computational power, a more realistic and accessible virtual scene with a lower perceivable delay is expected in the foreseeable future. Thus, XR technologies will be incorporated with more advanced techniques to further extend their capabilities. For example, a better mixture of virtual and real elements, such as texture, temperature, motion, and weight simulation, will make the perceivable details richer. On the other hand, these simulated features can be assembled in ways that will be impossible in the real world, allowing people to shuffle their fixated minds and broaden their ideation scope.

Mitigating conceptual blocks. The narrow mindset due to the priming effect, anchoring effect, and stereotypes often lead people to delimit problem areas in a confined manner, which is a major conceptual barrier in the ideation process. For this problem, XR technologies provide a unique solution by enabling operators to experience others' perspectives:

- Changing perspective. By providing high-level control of the interaction environment, XR technologies allow users to virtually simulate the world of others and see the world from their eyes. Research has already shown that VR can promote empathy and understanding while reducing stereotypes and incorrect knowledge of outgroup members [5]. Thus, people will be able to immerse themselves in different simulated environments and see the world through the eyes of a 
diverse group of people, such as the elderly, infants, athletes, and office staff. With the adoption of various viewpoints, the influence of stereotypes and fixated mindset on individuals can be alleviated and hence facilitate creative ideation. Universal design will particularly benefit from this possibility.

- Intuitive and natural operation. If a complex or introduction-required ideation technology is adopted, the information in tutorials may lead to mind fixation. For example, tutorials and guiding projects impose a deep impression on novice designers, making them fixate on the exposed features [6]. An advantage of XR's flexible and intuitive mode of operation is that it supports dealing with design situations in such a straightforward way that it does not require excessive guidance. This is because XR technologies intensively incorporate hand movements and gesture recognition during interactions. On the other hand, XR technologies impose minimal technologically relevant distractions so that people can focus on the ideation task itself.

\section{Mitigating ideation uncertainty}

blocks. The psychological burden of losing the invested effort and resources can easily drive people to reject unfamiliar and untested ideas and miss the chance for innovation. Fortunately, the easy recovery of previous working steps and the possibility of convenient virtual prototyping and testing in the XR environment can resolve this problem:

- Easy redo and undo. When a previous work in progress can be easily retrieved, the worries of wasting previous effort will be significantly relieved. However, most digital ideation tools achieve such a function at the cost of effortconsuming operation, which in turn facilitates mind fixation. By supporting intuitive interaction and the easy retrieval of previous work, $\mathrm{XR}$ technology can free designers from this trade-off and ensure a convenient recovery of the working steps.

- Fast prototyping. Fast prototyping is an ideation method that can counter mind fixation by reducing resources invested early in the ideation process. The principle of this strategy is to externalize the core of an idea with the least input, so that the feasibility of an idea can be assessed while avoiding fixation due to spent efforts. XR technologies strike an ideal balance between fidelity and reliability when conveying ideation outcomes. The easy construction of virtual objects in an XR environment allows operators to illustrate their ideas with minimal effort while ensuring that an idea can be demonstrated with adequate details.

- Convenient test and simulation. Ideation is not a linear process and often requires multiple iterations. If people can validate that an idea is promising, or at least plausible, they are more likely to avoid status-quo bias in the idea-generation process. By providing convenient prototyping, $\mathrm{XR}$ technologies are ideal for testing and simulating ideation outcomes.

\section{CONCLUSION}

With this discussion on the use of XR technologies in mitigating longstanding mind-fixation issues, we hope to pull the attention of researchers toward integrating novel technologies into the innovation and creativity research domains. The value of XR in these fields is still underrecognized. In the current practice, XR technologies are often used merely as fancy painting or modeling tools, while their potential in achieving breakthroughs in human-technology interaction has been largely overlooked. The challenges in human-technology interaction are often related to humans' inherent mental mechanisms and capabilities, which leaves limited space for solving these problems. By engaging a different set of cognitive and behavioral functions, XR technologies have pointed to an alternative strategy to address this dilemma: Instead of providing a solution, directly eliminating the problem may sometimes be more efficient and effective. Similar to mitigating the mind-fixation phenomenon by excluding fixation-prone mental processes, XR technologies have shown capabilities in overcoming more tricky problems in our interaction with this technologymediated world. With the rapid advancement in XR technologies, more long-persisting barriers may be addressed, with the possibility of taking human-technology interaction into a new realm.

\section{ENDNOTES}

1. Leung, A.K.Y., Kim, S., Polman, E., Ong, L.S., Qiu, L., Goncalo, J.A., and SanchezBurks, J. Embodied metaphors and creative "acts." Psychological Science 23, 5 (2012), 502-509.

2. Youmans, R. J. and Arciszewski, T. Design fixation: Classifications and modern methods of prevention. AI EDAM 28, 2 (2014), 129-137.

3. Viswanathan, V. and Linsey, J. Design fixation in physical modeling: an investigation on the role of sunk cost. Proc. of the ASME Design Engineering Technical Conference, 2011.

4. Yang, X., Lin, L., Cheng, P.Y., Yang, X., Ren, Y., and Huang, Y.M. Examining creativity through a virtual reality support system. Educational Technology Research and Development 66, 5 (2018), 1231-1254.

5. Hu, X., Georgiev, G.V., and Casakin, H. Mitigating design fixation with evolving extended reality technology: An emerging opportunity. Proc. of the Design Society: DESIGN Conference. 2020, 1305-1314.

6. Georgiev, G.V., and Milara, I.S. Idea generation challenges in digital fabrication. Proc. of the Fifth International Conference on Design Creativity. 2018, 85-92.

\footnotetext{
Xinhui Hu is a member of Georgi Georgiev's Design Research group. Her recent research interest is to adopt extended-reality technologies in supporting the design process, especially in bridging the cognitive gaps between technology usage and the natural flow of design.

$\rightarrow$ xinhui.hudoulu.fi
}

Vijayakumar Nanjappan received his Ph.D. in computer science with a specialization in human-computer interaction from the University of Liverpool, U.K., in 2020. He was a graduate researcher at X-CHI Lab from 2015 to 2020 . His research interests include $\mathrm{HCl}$, wearable devices, digital fabrication, and learning technologies.

$\rightarrow$ Vijayakumar.Nanjappandoulu.fi

Georgi Georgiev's work is focused on design creativity, digital fabrication, design cognition, idea generation, and design computing. He is an associate professor in design research and digital fabrication at the Center for Ubiquitous Computing (UBICOMP), University of Oulu, Finland.

$\rightarrow$ georgi.georgievdoulu.fi 